\title{
La posición de Trendelenburg no cambia el gasto cardíaco en voluntarios sanos
}

\author{
Trendelenburg position does not change Cardiac output \\ in healthy volunteers
}

Alejandro Jankelevich¹, M. Carolina Cabrera¹, Camila González² , Francisca Castillo', Jaime de la Maza

\begin{abstract}
Background: The Trendelenburg position (TP) has been empirically used to increase preload and thus cardiac output (CO). Transthoracic echocardiogram (TTE) is a noninvasive technique that estimates $C O$. We aimed to describe if the TP can improve CO. Materials and Methods: We conducted a descriptive study in healthy subjects (ASA I), with TTE measurements of CO and the End-Diastolic Volume of the Left Ventricle (EDVLV) in supine and after 5 minutes in TP. Results: There showed no significant changes in blood pressure, heart rate and pulse oximetry in $0^{\circ}$ and after 5 minutes in $45^{\circ}$. The mean $\mathrm{CO}$ without inclination was 4.2 $1 / \mathrm{min} \pm 0.6$, tat is the same results after 5 minutes in PT $(p>0.6)$. The EDVLV was $95 \pm 12 \mathrm{ml}$ at $0^{\circ}$ and $101 \pm 15 \mathrm{ml}$ after 5 minutes in PT $(p>0.8)$. Conclusion: Trendelenburg position doesn't improve CO or preload in healthy subjects.
\end{abstract}

\section{RESUMEN}

Introducción: Empíricamente se ha utilizado la posición de Trendelenburg (PT) para aumentar la precarga y posteriormente el gasto cardiaco (GC). La ecografía transtorácica (ETT) es una técnica no invasiva que puede estimar el GC. Realizamos un estudio descriptivo para evaluar si la PT afecta el CG. Materiales y Métodos: Examinamos pacientes ASA I, se hicieron mediciones del GC y del volumen de fin de diástole (VDFVI) en posición supina y luego de cinco minutos en PT. Resultados: No se observaron cambios significativos en la presión arterial, frecuencia cardíaca y saturometría en los voluntarios a $0^{\circ}$ y a $45^{\circ}$. El GC sin inclinación fue en promedio 4,2 l/min $\pm 0,6$, el mismo resultado tras cinco minutos en PT, sin ser estadísticamente significativo $(p>0,6)$. El VDFVI a $0^{\circ}$ fue en pro-

\section{Key words:}

Trendelenburg, cardiac output, transthoracic echocardiogram

\section{Palabras clave:}

Trendelenburg, gasto cardiaco, ecografía transtorácica

Departamento de Anestesiología Universidad de Valparaíso, sede Hospital Clínico FACH.

Anestesiólogo Clínica Dávila.

Fecha de recepción: 10 de junio de 2018

Fecha de aceptación: 26 de febrero de 2019

\section{ORCID}

https://orcid.org/0000-0003-1524-5812

\section{Correspondencia:}

Dr. Alejandro Jankelevich

Email: janke24@gmail.com 
medio $95 \pm 12 \mathrm{ml}$ y tras cinco minutos en PT $101 \pm 15 \mathrm{ml}$, que tampoco alcanzó diferencia estadísticamente significativa $(p>0,8)$. Conclusión: La posición de Trendelenburg no varía el gasto cardiaco ni la precarga en voluntarios sanos.

\section{Introducción}

E I manejo de la volemia es un tema largamente discutido y de gran importancia en la anestesiología. La perfusión adecuada de los tejidos permite la entrega de nutrientes para el metabolismo celular y la remoción de los productos del metabolismo. El gasto cardiaco (GC) juega un rol importante en la perfusión de órganos. Empíricamente se ha utilizado la posición de Trendelenburg (PT) para desviar la sangre desde las extremidades inferiores hacia la circulación central, aumentando así la precarga y por el mecanismo de frank-starling, el volumen sistólico[1]-[6]. La ecocardiografía transtorácica (ETT) es una técnica no invasiva que permite la estimación de gasto cardíaco y volemia. El sistema venoso es complejo y está regulado por receptores simpáticos[7], por lo que es posible que la simple desviación de sangre por gravedad no sea suficiente para aumentar la precarga.

Dado que la PT se ha utilizado de forma empírica, es importante evaluar si realmente afecta el GC y ser finalmente validada como una herramienta de reanimación hemodinámica.

\section{Materiales y Métodos}

Se realizó un estudio descriptivo, con 78 voluntarios de 20 a 39 años, 44 hombres, ASA I, sin ayuno. Los voluntarios fueron monitorizados de manera no invasiva con ECG, saturometría y PANI. La ETT fue rea-

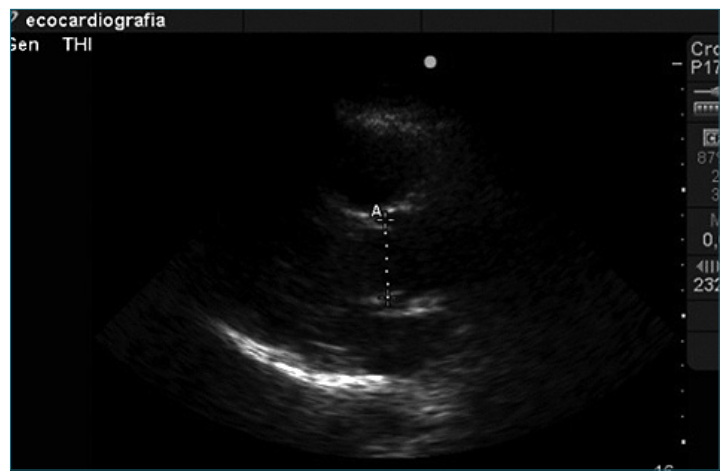

Figura 1. Medición del diámetro del tracto de salida. lizada por un anestesiólogo entrenado con transductor cardíaco.

Nuestro objetivo primario fue evaluar cambios en el GC tras cinco minutos en PT y el objetivo secundario fue evaluar cambios en el volumen de fin de diástole (VDFVI) en PT.

Las imágenes obtenidas fueron las siguientes:

Primero desde ventana paraesternal en eje largo se midió el diámetro del tracto de salida. Este se dividió

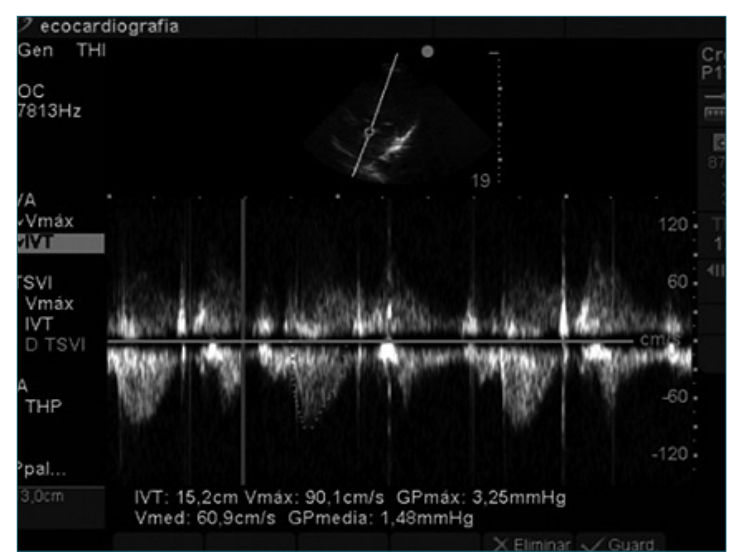

Figura 2. Doppler continuo a nivel del tracto de salida para medición del IVT.

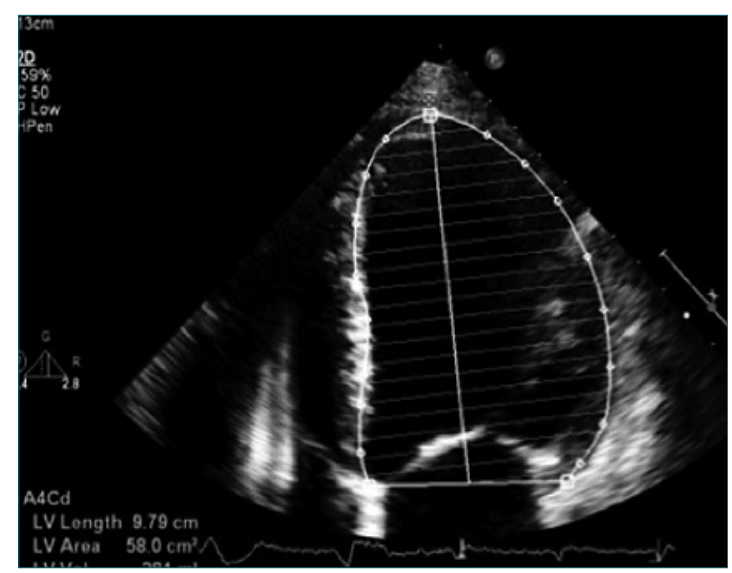

Figura 3. Cálculo de la Fracción de eyección del VI con técnica de Simpson 
en dos para obtener el radio $(3,14 \times$ r 2), correspondió al área valvular aórtica (Figura 1).

Luego desde ventana apical se estimó la integral de la velocidad en el tiempo (IVT) del tracto de salida (Figura 2). Al multiplicar el área x IVT x FC = GC. Cada una de las mediciones fue realizada en decúbito supino sin inclinación y luego tras cinco minutos en PT. El VFDVI se midió en 4 cámaras (Figura 3).

El análisis estadístico fue realizado con STATA 10.0, valor-p $<0,05$ para significancia estadística.

\section{Resultados}

La edad promedio de los voluntarios fue $32 \pm$ 6 años. Las ventanas ecocardiográficas obtenidas permitieron realizar las mediciones en todos los casos. No se observaron cambios significativos en la presión arterial, frecuencia cardíaca y saturometría en los voluntarios a $0^{\circ}$ y a $45^{\circ}$. El GC promedio fue $4,21 / \min \pm 0,6$ en ambos grupos con un valor $p>$ 0,6 . El VDFVI a $0^{\circ}$ fue en promedio $95 \pm 12 \mathrm{ml}$ y tras cinco minutos en PT $101 \pm 15 \mathrm{ml}$, con valor $p$ $>0,8$. (Figuras 4,5 y 6).

Tanto el resultado primario como el secundario no alcanzaron valores estadísticamente significativos. Por lo tanto, en sujetos jóvenes, sanos, en estado de normovolemia y sin anestesia, el GC y el volumen de fin de diástole no sufren cambios importantes tras cinco minutos de PT.

\section{Discusión}

El sistema venoso contiene el $70 \%$ del volumen sanguíneo total y es 30 veces más complaciente el sistema arterial. Es por esto que se habla del sistema venoso como un sistema de "reserva" de sangre que fácil e inmediatamente traspasa su volumen para mantener la presión de llenado del corazón derecho[7].

Las venas esplácnicas y cutáneas son más complacientes que las venas en las extremidades inferiores. Las venas esplácnicas y cutáneas tienen receptores a1 y a2 y son sensibles a la estimulación simpática[7].

Existen dos tipos de volúmenes a nivel venoso: estresado y no estresado. El volumen estresado es el volumen que determina la presión de llenado circulatoria media y afecta directamente el retorno venoso y el GC. El volumen no estresado es el volumen que puede ser movilizado a la circulación cuando es necesario[7],[8]. En situación de necesidad el sistema complaciente (esplácnico) aumenta la precarga y directamente, por la ley de Frank - Starling, aumenta el GC. Por otro lado, se sabe que la transferencia pasiva de sangre venosa es más importante que la transferencia activa por venoconstricción[7],[8].

Durante la anestesia se pierde la regulación simpática (parcial o completamente), esta pérdida de la actividad simpática venodilata el sistema esplácnico y así disminuye la precarga y el GC[8]-[10].

En nuestro trabajo se demuestra que en pacientes

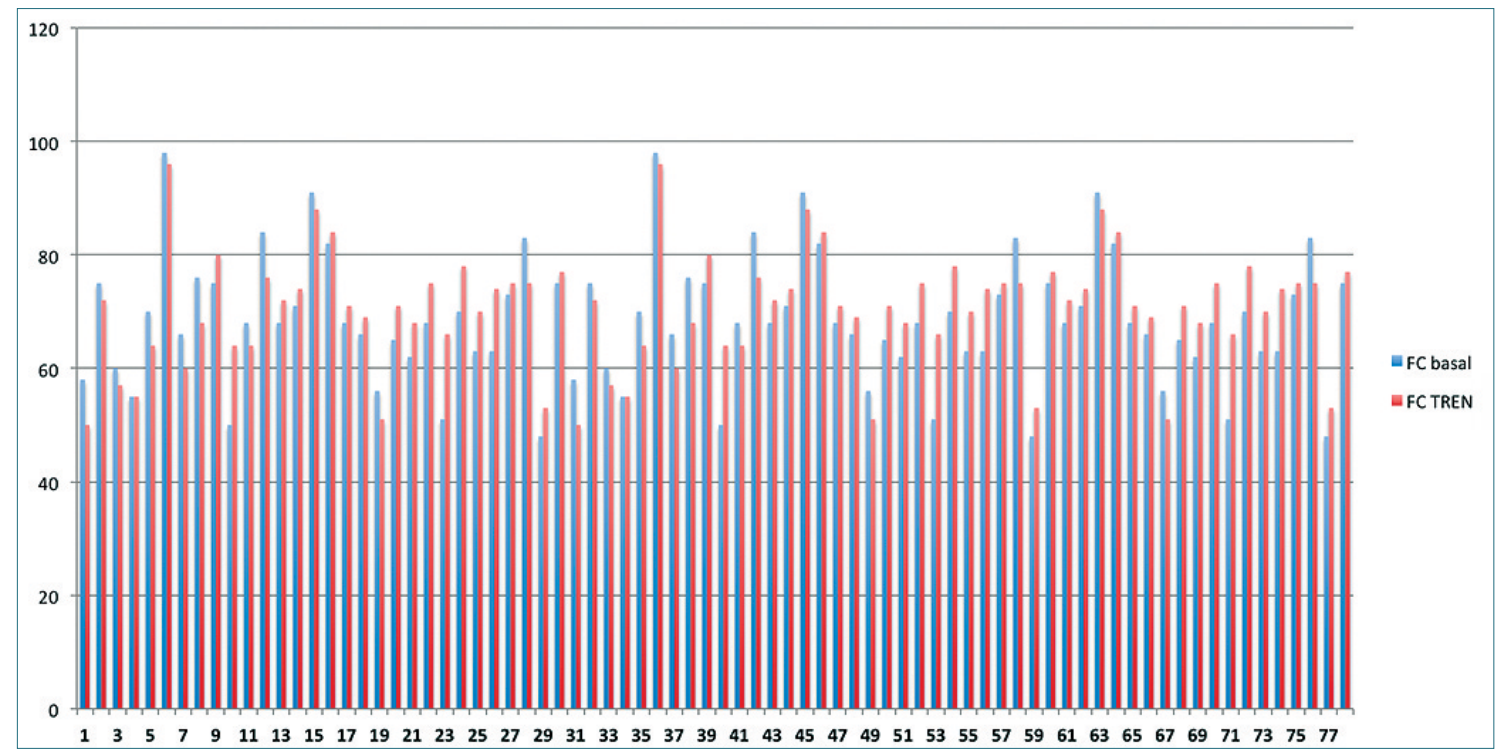

Figura 4. Frecuencia cardíaca observada previo y posterior a la posición de Trendelemburg. 


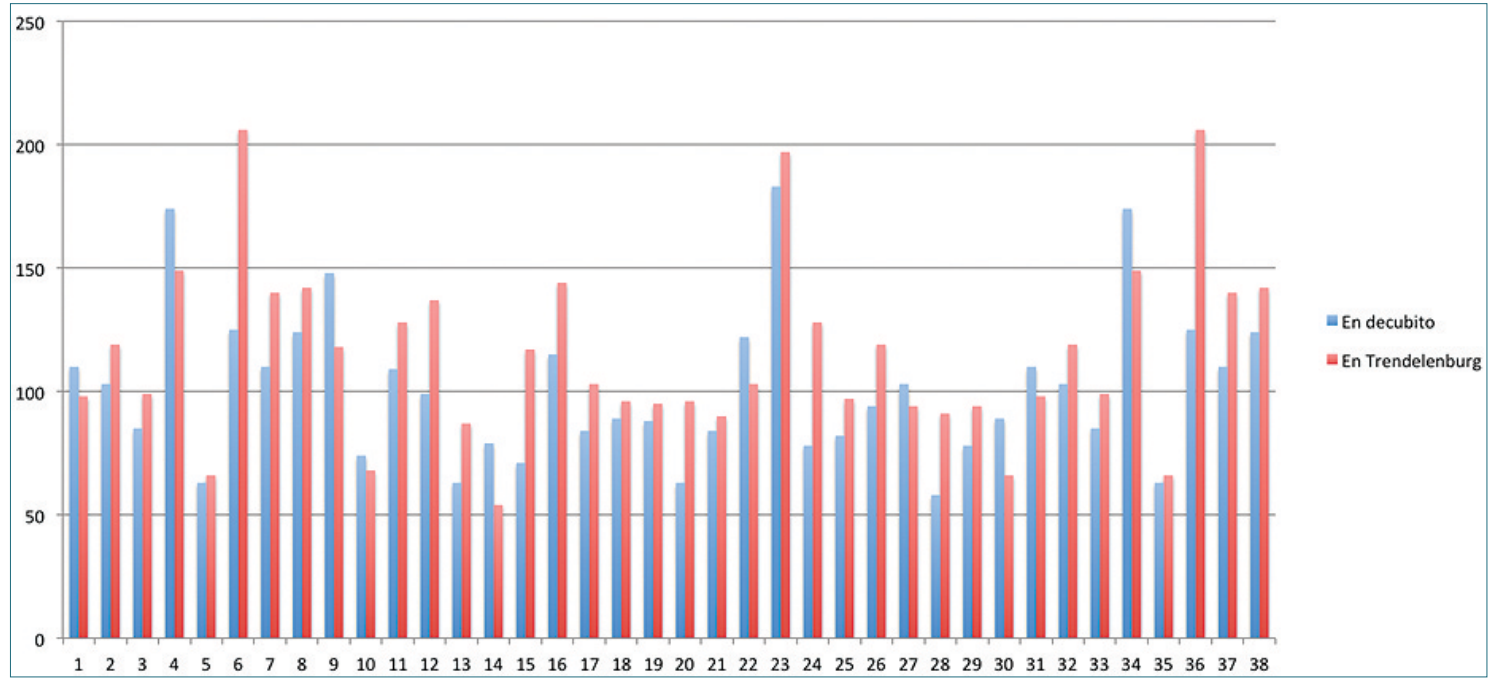

Figura 5. Volumen de fin de diástole previo y posterior a la posición en Trendelemburg.

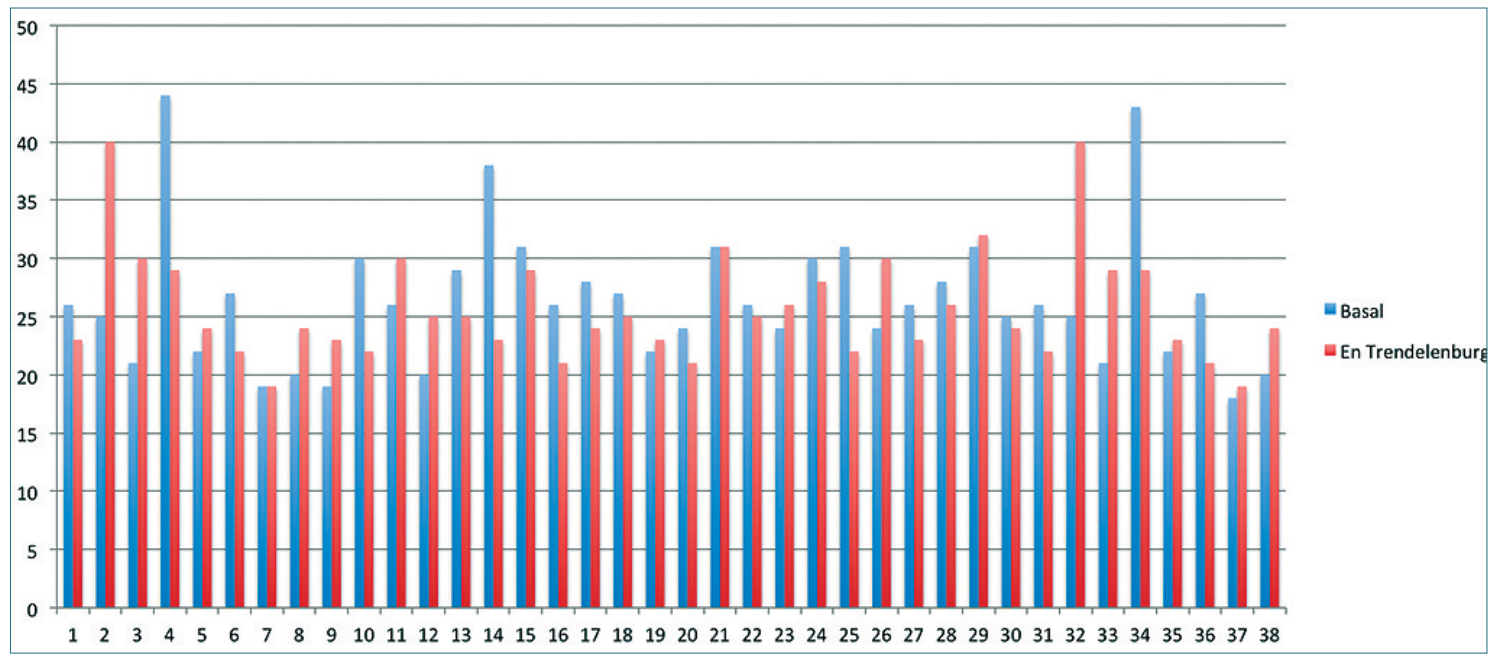

Figura 6. Integral de velocidad sobre tiempo (IVT) previo y posterior a la posición de Trendelemburg.

sanos, jóvenes y despiertos los cambios de posición no afectan la presión arterial, GC o PVC. Esto posiblemente ocurre porque la activación inmediata de reflejos simpáticos, además del sistema renina angiotensina aldosterona, liberan vasoconstrictores aumentando la precarga y el GC.

Es de esperar que la relación entre la volemia y cambios de posición sea diferente en pacientes sometidos a anestesia general, regional o en estado de hipovolemia dado a los cambios fisiológicos que ocurren en estos pacientes. Dado que, en algunos estudios la maniobra de Trendelenburg ha demostrado aportar entre 150 - $300 \mathrm{cc}$ al sistema circulatorio central, durante los últimos años se ha investigado la utilidad de esta maniobra en pacientes que reciben anestesia para cirugía cardiaca[11] y en pacientes que estén en el departamento de urgencias para saber si estos serían respondedores de volumen[12],[13]. Por ahora, los resultados aún son inciertos e insuficientes para recomendar la posición de Trendelenburg. Estos resultados tampoco permiten recomendar la PT para situaciones extremas, como el paro cardiorrespiratorio[14],[15]. 


\section{Conclusión}

La posición de Trendelenburg no varía el gasto cardiaco ni la precarga en voluntarios sanos, jó- venes y despiertos. Aún se debe extender el estudio en pacientes con patologías de base, anestesiados e hipovolémicos para finalmente poder descartar o recomendar esta maniobra en situaciones especiales.

\section{Referencias}

1. Bivins HG, Knopp R, dos Santos PA. Blood volume distribution in the Trendelenburg position. Ann Emerg Med. 1985 Jul;14(7):6413. https://doi.org/10.1016/ S0196-0644(85)80878-7 PMID:4014811

2. Jiménez EJ. Modulating the response to injury (initial management for hypotension). In: Civetta JM, Taylor RW, Kirby RR, editors. Critical Care. 3rd ed. Philadelphia $(\mathrm{Pa})$ : Lippincott Williams \& Wilkins; 1997. pp. 374-5.

3. Hofer CK, Zalunardo MP, Klaghofer R, Spahr T, Pasch $T$, Zollinger $A$. Changes in intrathoracic blood volume associated with pneumoperitoneum and positioning. Acta Anaesthesiol Scand. 2002 Mar;46(3):303-8. https://doi. org/10.1034/j.1399-6576.2002. t01-1-460313.x PMID:1 1939922

4. Joris JL, Noirot DP, Legrand MJ, Jacquet NJ, Lamy ML. Hemodynamic changes during laparoscopic cholecystectomy. Anesth Analg. 1993 May;76(5):1067-71. https:// doi.org/10.1213/00000539199305000-00027 PMID:8484509

5. Odeberg $S$, Ljungqvist $O$, Svenberg T, Gannedahl P, Bäckdahl $M$, von Rosen A, et al. Haemodynamic effects of pneumoperitoneum and the influence of posture during anaesthesia for laparoscopic surgery. Acta Anaesthesiol Scand. 1994 Apr;38(3):276-83. https://doi. org/10.1111/j.1399-6576.1994. tb03889.x PMID:8023669

6. Hirvonen EA, Nuutinen LS, Vuolteenaho $\mathrm{O}$. Hormonal responses and cardiac filling pressures in head-up or head-down position and pneumoperitoneum in patients undergoing operative laparoscopy. $\mathrm{Br} J$ Anaesth. 1997 Feb;78(2):128-33. https:// doi.org/10.1093/bja/78.2.128 PMID:9068326

7. Rothe CF. Reflex control of veins and vascular capacitance. Physiol Rev. 1983 Oct;63(4):1281342. https://doi.org/10.1152/ physrev.1983.63.4.1281 PMID:6361810

8. Hainsworth R. The importance of vascular capacitance in cardiovascular control. News Physiol Sci. 1990;5(6):2504. https://doi. org/10.1152/physiologyonline.1990.5.6.250.

9. Edouard DA, Pannier BM, London GM, Cuche JL, Safar ME. Venous and arterial behavior during normal pregnancy. Am J Physiol. 1998 May;274(5 Pt 2):H1605-12. PMID:9612370

10. Gelman S. Venous function and central venous pressure: a physiologic story. Anesthesiology. 2008 Apr;108(4):73548. https://doi.org/10.1097/ ALN.0b013e3181672607 PMID:18362606

11. Fakhari $S$, Bilehjani $E$, Farzin $H$, Pourfathi H, Chalabianlou M.
The effect of passive leg-raising maneuver on hemodynamic stability during anesthesia induction for adult cardiac surgery. Integr Blood Press Control. 2018 Jun;11:57-63. https:// doi.org/10.2147/IBPC.S126514 PMID:29922085

12. Duus N, Shogilev DJ, Skibsted S, Zijlstra HW, Fish E, Oren-Grinberg A, et al. The reliability and validity of passive leg raise and fluid bolus to assess fluid responsiveness in spontaneously breathing emergency department patients. J Crit Care. 2015 Feb;30(1):217.e1-5. https://doi. org/10.1016/j.jcrc.2014.07.031 PMID:25262530

13. Cherpanath TG, Hirsch $A$, Geerts BF, Lagrand WK, Leeflang MM, Schultz MJ, et al. Predicting fluid responsiveness by passive leg raising: a systematic review and meta-analysis of 23 clinical trials. Crit Care Med. 2016 May;44(5):98191. https://doi.org/10.1097/ CCM.0000000000001556 PMID:26741579

14. Bridges $N$, Jarquin-Valdivia AA. Use of the Trendelenburg position as the resuscitation position: to T or not to T? Am J Crit Care. 2005 Sep;14(5):364-8. PMID:16120887

15. Peña B. Rodríguez Larrad A. Does the Trendelenburg position affect hemodynamics? A systematic review. Emergencias. 2012;24:143-50. 